\title{
Paroxysmal atrial fibrillation: Scuba diving or hyperbaric oxygen therapy
}

\author{
Raja Ben Sassi ${ }^{2}$, Karima Taamallah ${ }^{1,3}$, Imen Mezoughi ${ }^{2}$, Walid Belhaj Brahim ${ }^{2}$, Zied Hajjej $^{1,4}$, \\ Hedi Gharsallah ${ }^{1,2}$
}

${ }^{1}$ Université de Tunis El Manar, Faculté de Médecine de Tunis, 1007, Tunis, Tunisie

${ }^{2}$ Hôpital militaire de Tunis, centre d'oxygénothérapie hyperbare, 1008, Tunis, Tunisie

${ }^{3}$ Hôpital militaire de Tunis, service de chirurgie cardiaque, 1008, Tunis, Tunisie

${ }^{4}$ Hôpital militaire de Tunis, service d'anesthésie réanimation, 1008, Tunis, Tunisie

\author{
Email address: \\ gharsallahhedi@gmail.com (H. Gharsallah)
}

\section{To cite this article:}

Raja Ben Sassi, Karima Taamallah, Imen Mezoughi, Walid Belhaj Brahim, Zied Hajjej, Hedi Gharsallah. Paroxysmal Atrial Fibrillation: Scuba Diving or Hyperbaric Oxygen Therapy. American Journal of Sports Science. Vol. 2, No. 5, 2014, pp. 138-140.

doi: 10.11648/j.ajss.20140205.16

\begin{abstract}
Heart rhythm disorders are widely reported as complications of scuba diving but rarely after HBO therapy. We report the case of a professional diver who developed a paroxysmal atrial fibrillation after hyperbaric oxygen therapy as treatment of diving accident. It is a professional diver admitted for the treatment of a cochleovestibular desaturation accident. The initial ECG showed normal sinus rhythm. He received 10 HBO therapy sessions. We discovered a heart rhythm disorder. The ECG showed an atrial fibrillation. Heart rhythm returned to sinus after receiving FLECAIN ${ }^{\circ}$. Control ECG after 2 months showed a normal sinus rhythm.
\end{abstract}

Keywords: Atrial Fibrillation - HBO Therapy - Scuba Diving

\section{Introduction}

Scuba diving and diving in a hyperbaric chamber are two conditions that expose to the complications of overpressure. Heart rhythm disorders are widely reported as complications of scuba diving but rarely after HBO therapy.

We report the case of a professional diver who developed a paroxysmal atrial fibrillation after hyperbaric oxygen therapy as treatment of a diving accident.

\section{Case Description}

Our patient is a 33 years old professional diver admitted for the treatment of a cochleovestibular desaturation accident due to a 30 minutes dive at 60 meters depth. While emerging out of water he presented rotary vertigo which became disabling when he arrived to the surface. Clinical examination at the hyperbaric oxygen therapy center upon admission found a patient presenting a serious vertigo crisis with no other neurological localization signs. Eardrum examination was normal. Initial heart auscultation was normal and ECG showed normal sinus rhythm (fig 1A).

Treatment in our center consisted in a first HBO emergency recompression session at $18 \mathrm{~m}$ (2.8ATA) that improved partially the symptoms followed by 10 consolidation sessions. The outcome was favorable: disappearance of the vertigo at the end of treatment. Cerebro- medullary MRI showed no abnormalities. While performing a transthoracic echocardiography (in search of patent foramen ovale), we discovered a heart rhythm disorder. ECG showed an atrial fibrillation (fig 1B). The rhythm returned to normal after receiving FLECAIN®. No patent foramen ovale was found in transoesophagal echocardiography. The patient was advised not to undertake diving activities any more. Control ECG after 2 months showed a normal sinus rhythm (fig 1C). 

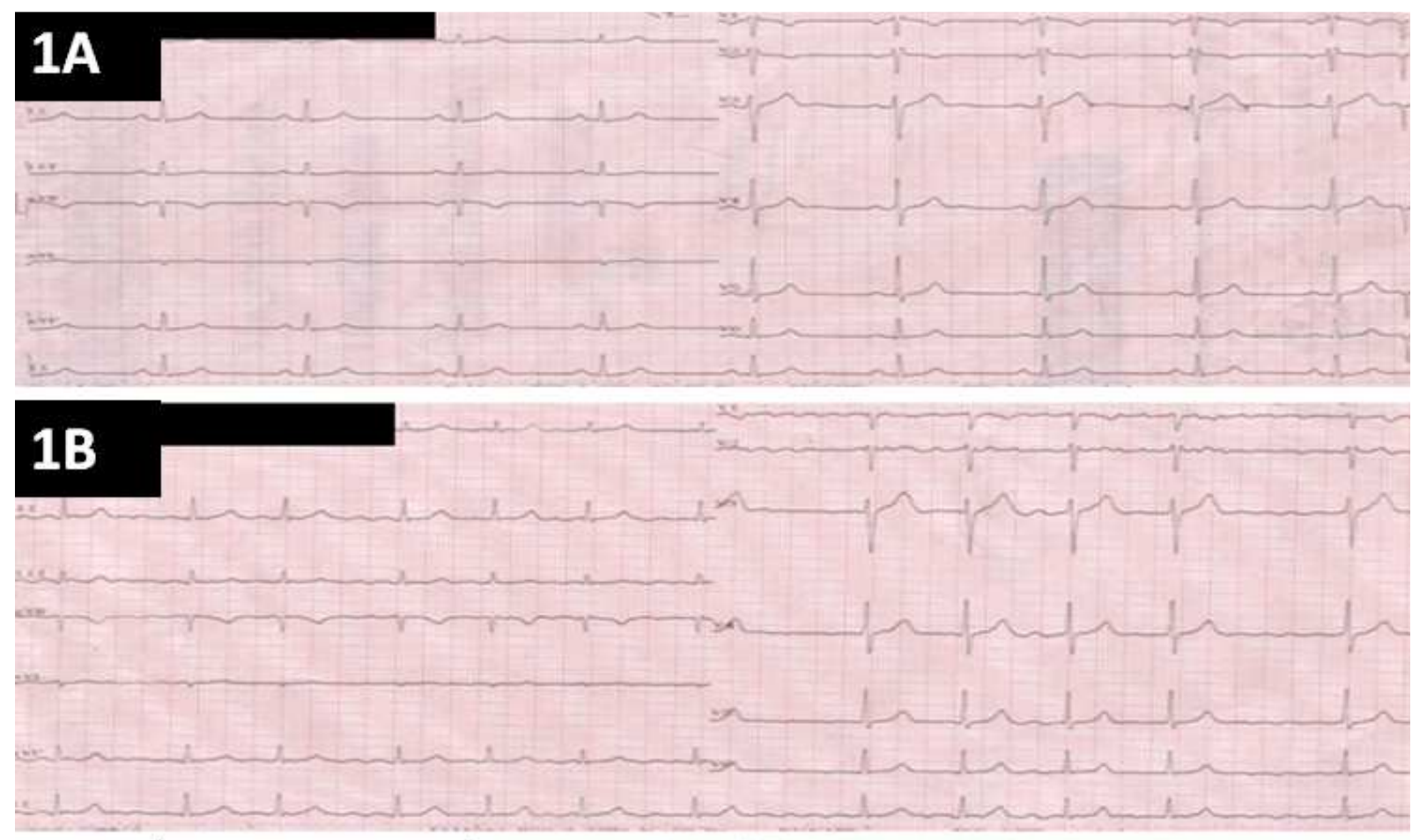

\section{$1 C$}

Fig 1. Patient's electrocardiograms: 1A: Before HBO therapy, 1B: Atrial fibrillation after 10 sessions of HBO therapy, 1C: after 2months of follow up: return to sinus rythm

\section{Discussion}

Paroxysmal atrial fibrillation is the most frequent supraventricular heart rhythm disorder. It is a high risk condition for thromboembolic disease. Valvular disease and myocardial injury are usually the underlying causes. In scuba diving, paroxysmal atrial fibrillation is associated to two physiopathological mechanisms. The first is mechanical related to the high pressure while emerging under water. The second is nervous via the activation of skin thermo receptors.It involves also other conditions: temperature of water, diving procedures and diving suit. In fact, conflict between the two components of the autonomic nervous system; bradycardia resulting from parasympathetic stimulation during diving on one hand and tachycardia due to sympathetic stimulation of the skin thermo receptors on the other hand, can lead to heart rhythm disorders. Some risk factors predispose to heart rhythm disorders: hereditary or acquired syndrome of long QT, hypertensive cardiomyopathy, stress and tiredness (1).
Schipke et al. showed that scuba diving causes psychological stress (2).

Our patient had no cardiovascular risk factors, transoesophagal echocardiography showed no PFO. Nevertheless, he reported that the water was quiet cold and he was exhausted from daily diving session during the last month.

Hyperbaric oxygen therapy is a treatment procedure that consists in giving the patient oxygen at a pressure higher than the level of atmospheric pressure allowing a hyper oxygenation of the tissues. Diving accident is a type 1 recommendation (second EUROPEAN consensus conference on the treatment of decompression illness from recreational diving. Marseille, May 9 to 11, 1996).The complications of HBO treatment are mostly barotraumas of the medial ear, the sinus or the lungs and toxicity due to hyperoxia. Clinical signs of hyperoxia are generally neurological. Cardiac impact is not described in literature. In fact, only one author published the occurrence of paroxysmal atrial fibrillation after a HBO session in a carbon monoxide poisoning case. He supposed that 
inhibition of the conduction in the atrial sinus due to hyperoxigenation led to this heart rhythm disorder (3). This case report has raised a serious controversy (4). This complication appeared after a variable period of time. Our patient was initially asymptomatic. His arrhythmia was discovered accidently after the end of the sessions. Therefore we cannot precise the exact moment of happening of this trouble. The rhythm returned to sinus after oral intake of FLECAIN ${ }^{\circledR}$. No ECG abnormalities were detected during the follow up period.

In our case, we cannot dismiss two etiologies. The first one is scuba diving but initial ECG showed regular sinus rhythm. The second etiology is HBO treatment since it is closely related to the appearance of this arrhythmia especially as echocardiography showed no organic abnormalities. No distant recurrence was detected because there was no more exposure to scuba diving or HBO treatment.

Finally, two questions need to be answered:

1) Is ECG monitoring interesting for the patients before and after HBO sessions?

2) Can this patient take up scuba diving again?

\section{Conclusion}

Although Cardiac rhythm disorder due to scuba diving is a plausible hypothesis, the role played by HBO therapy in the genesis of this trouble cannot be discarded. ECG monitoring can be useful before and after HBO therapy sessions.

\section{References}

[1] M.J. Schattock, M.J. Tipton. Autonomic conflict: a different way to die during cold water immersion? J. Physiol 2012; 590(14 ):3219-3230.

[2] J.D. Schipke, M. Pelzer. Effect of immersion, submersion, and scuba diving on heart rate variability. Br. J. Sports Med $2001 ; 35: 174-180$.

[3] G. Celbek, B.V. BOZ. Paroxysmal atrial fibrillation after hyperbaric oxygen therapy. Am J Emerg Med 2013;31:261.e5 - 261.e7.

[4] N. Zamani. The cause of Paroxysmal atrial fibrillation: hyperbaric oxygen therapy or carbon monoxide poisoning. Am J Emerg Med. 2013;31(1):247. 